\section{Evolución prolongada en síndrome de Crigler-Najjar tipo I}

\author{
SANTIAGO BESAa, CARLOS I. CALVO ${ }^{a}$, PAUL R. HARRIS
}

\section{Crigler-Najjar syndrome. Report of one case with a long term follow up}

Crigler-Najjar Syndrome is an uncommon genetic disorder characterized by the elevation of unconjugated plasmatic bilirubin secondary to deficiency of the enzyme uridine diphosphate glucuronyltransferase (UDP-GT). We report a 19-years-old woman with the syndrome diagnosed during the neonatal period, when she developed a severe jaundice in the first 10 days of life, reaching unconjugated bilirubin levels of $29 \mathrm{mg} / \mathrm{dl}$, with normal liver function tests. After transient response to phototherapy, the patient was referred to a tertiary medical center in which an extensive work up ruled out other etiologies and the diagnosis of type I Crigler-Najjar syndrome was established. Currently, the patient has a mild mental retardation. She is receiving homemade phototherapy $18 \mathrm{~h}$ per day with acceptable control of bilirubin levels. Many mutations have been associated with UDP-GT dysfunction resulting in a broad spectrum of the disease. When bilirubin rises above physiological limits, it permeates the hematoencephalic barrier, inducing bilirubin impregnation of basal ganglia with secondary neuronal damage and necrosis. The worst outcome, kernicterus, is characterized by mental retardation, central deafness, ophthalmoplegia, ataxia, athetosis, spasticity, seizures and death. First line therapy includes phototherapy, but definitive therapy is liver transplantation before the occurrence of neurological damage.

(Rev Med Chile 2014; 142: 109-113)

Key words: Crigler-Najjar Syndrome; Jaundice; Phototherapy.

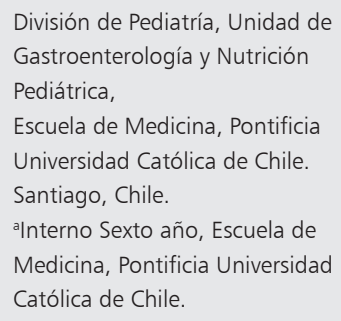

E 1 síndrome de Crigler-Najjar (SCN) es una entidad nosológica infrecuente, con una frecuencia estimada de 0,6 por millón de habitantes ${ }^{1}$. Se describió por primera vez en 1952 por dos pediatras, en 6 niños que desarrollaron ictericia grave en los primeros días de vida, falleciendo por kernicterus antes de los 2 años de edad'.

El SCN es un trastorno del metabolismo de la bilirrubina (Figura 1) generado por un déficit de la enzima uridindifosfato-glucoroniltransferasa (UDP-GT), de forma total (SCN tipo I) o parcial (SCN tipo II), generando un aumento de la bilirrubina indirecta plasmática ${ }^{3}$. Este defecto se hereda de manera autosómica recesiva por mutaciones en el gen UGT1A1 del cromosoma 2, el mismo gen implicado en el síndrome de Gilbert. Se presenta como hiperbilirrubinemia indirecta con pruebas hepáticas normales y sin signos de hemólisis ${ }^{4}$. En el SCN tipo I la bilirrubina indirecta (BI) supera los $20 \mathrm{mg} / \mathrm{dl}$, casi no hay bilirrubina directa en la bilis y la respuesta al tratamiento con inductores de la enzima UDP-GT como el fenobarbital es casi nula ${ }^{5}$. Su principal complicación es el kernicterus $^{2}$, entidad anátomo-patológica caracterizada por necrosis en los ganglios de la base, hipocampo, núcleo cerebeloso y sustancia gris, producido por el paso de BI no unida a albúmina a través de la barrera hematoencefálica y su posterior depósito, alterando el metabolismo celular. . El kernicterus se manifiesta como sordera central, oftalmoplejia, ataxia, coreoatetosis, retraso mental, convulsiones, espasticidad y muerte ${ }^{6}$. El tratamiento consiste 


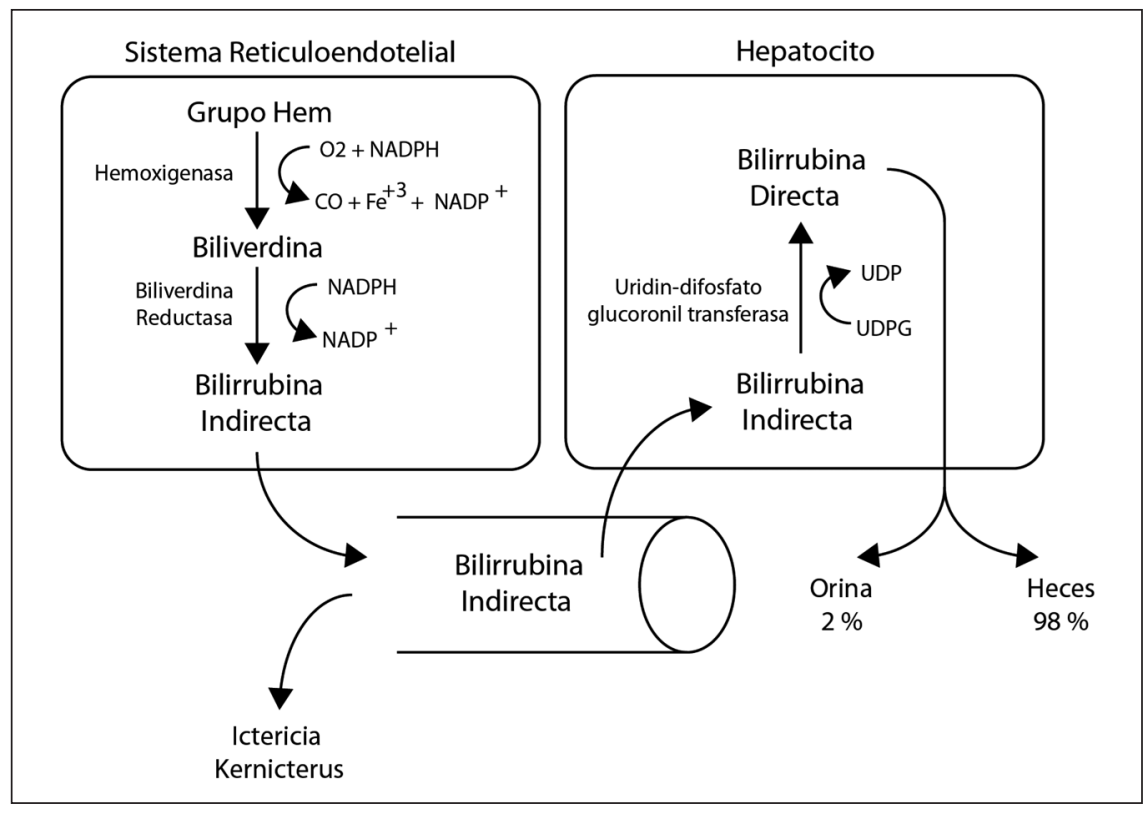

Figura 1. Metabolismo de la Bilirrubina, destacando la degradación del grupo Hem en la formación de bilirrubina indirecta y acumulación secundaria al déficit de la enzima uridindifosfato-glucoroniltransferasa (UDPG), generando un aumento de la bilirrubina indirecta plasmática. en fototerapia, que convierte la BI en un isómero excretable por la bilis. Se pueden asociar quelantes de sales biliares como colestiramina y sales cálcicas. En situaciones extremas se podría usar plasmaferesis como medida de salvataje, siendo el trasplante hepático el único tratamiento curativo ${ }^{4}$.

A pesar del esfuerzo terapéutico, la mayor parte de los niños fallecen en la primera década de la vida por complicaciones infecciosas y/o pulmonares asociadas al daño neurológico del kernicterus. Existen poco casos de sobrevida en adultos jóvenes. Una encuesta multicéntrica de tratamiento del SNC tipo I, reportó un paciente que murió a los 28 años de edad ${ }^{1}$. Existen escasos reportes de pacientes que alcanzaron la tercera década de vida ${ }^{7}$. El objetivo de este reporte es notificar el caso clínico único en Chile de un adulto joven sobreviviente de SCN tipo I con tratamiento médico.

\section{Caso clínico}

Paciente mujer de 19 años con antecedentes de síndrome de Crigler-Najjar tipo I diagnosticado al mes de vida. Fue primera hija de madre sana, padres no consanguíneos. El embarazo fue fisiológico. Nació de 38 semanas, parto eutócico y peso adecuado a edad gestacional (3.500 g).

La niña fue dada de alta al tercer día de vida en buenas condiciones generales, levemente ictérica y reingresada al $10^{\circ}$ día por ictericia acentuada con $19 \mathrm{mg} / \mathrm{dl}$ de bilirrubina total, que respondió a 7 días de fototerapia. Al mes de vida fue hospitalizada nuevamente para estudio por ictericia progresiva con bilirrubina total de hasta $29 \mathrm{mg} / \mathrm{dl}$, con 1,3 mg/dl de bilirrubina directa. Las pruebas hepáticas estuvieron dentro de rangos normales para la edad (SGOT $48 \mathrm{UI} / \mathrm{L}$, SGPT 56 $\mathrm{UI} / \mathrm{L}$, fosfatasas alcalinas $567 \mathrm{UI} / \mathrm{L}$ ) así como el hemograma (hematocrito $43 \%$, hemoglobina 14 $\mathrm{g} / \mathrm{dl}$, glóbulos blancos $2.200 \mathrm{x} \mathrm{mm}$, velocidad de sedimentación $12 \mathrm{~mm} / \mathrm{h}$ ). La ecografía abdominal mostró leve hepatomegalia, sin dilatación de la vía biliar. Fue trasladada a Santiago con la sospecha de una atresia de vías biliares.

Durante su estadía en el centro terciario, la niña fue evaluada con hemograma (hematocrito $40 \%$, hemoglobina $12,8 \mathrm{~g} / \mathrm{dl}$, glóbulos blancos 11.800

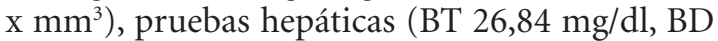
3,11 mg/dl, SGPT $19 \mathrm{UI} / \mathrm{L}$, fosfatasas alcalinas 267 UI/L, albúmina 4,4 g/L), TSH 4,8 UI/ml y T4 $12,6 \mathrm{mg} / \mathrm{dl}$. No hubo hallazgos anormales en la ecografía abdominal. Por tratarse de una ictericia de predominio indirecto, se comenzó tratamiento con fenobarbital $5 \mathrm{mg} / \mathrm{kg} /$ día, sin respuesta clínica ni de laboratorio. Se subió dosis de fenobarbital a $6,5 \mathrm{mg} / \mathrm{kg} /$ día, también sin respuesta. 
Se concluyó que la paciente presentaba el síndrome de Crigler-Najjar tipo I y se inició fototerapia nocturna a permanencia, logrando niveles de bilirrubina total cercanos a $11 \mathrm{mg} / \mathrm{dl}$ (con BD cercana a 1,5 mg/dl). Se trasladó a su hospital de origen para continuar con fototerapia y enseñar fototerapia artesanal nocturna en domicilio con luz blanca fluorescente (6-8 tubos), expuesta a 30-50 cm del niño por $18 \mathrm{~h}$ diarias.

La paciente evolucionó bien, con buen incremento pondo-estatural y desarrollo psicomotor. A los 2 años comenzó a presentar cuadros convulsivos a repetición, relacionados a una suspensión de la fototerapia por aproximadamente 2 meses. Las crisis fueron de difícil manejo, tratándose con fenobarbital y agregándose ácido valproico posteriormente. Desde el punto de vista cognitivo, la paciente presentó un retardo mental leve diagnosticado mediante el test de inteligencia para prescolares de Weschler (WISP) y la evaluación neurológica concluyó la presencia de kernicterus.

Los niveles de bilirrubina logrados con la fototerapia nocturna fueron en aumento a través de los años. Actualmente, la paciente se mantiene en fototerapia por $18 \mathrm{~h}$ diarias, logrando niveles de bilirrubina total que oscilan entre los 20 y 26 $\mathrm{mg} / \mathrm{dl}$. Se encuentra con $100 \mathrm{mg}$ de fenobarbital cada 12 h, sin crisis convulsivas desde hace 4 años.

\section{Discusión}

Este estudio reporta un caso único como es la sobrevida en un adulto joven con SCN tipo I tratado con fototerapia domiciliaria. El SCN es un diagnóstico que los pediatras y gastroenterólogos pediátricos deben tener en consideración al momento de enfrentarse a un neonato con ictericia prolongada, especialmente si no presenta hemólisis y los diagnósticos más comunes han sido descartados. Sin embargo, rara vez este es un diagnóstico diferencial para el gastroenterólogo de adultos, por la corta sobrevida de los pacientes.

Las mutaciones en el gen UGT1A1, en cualquiera de sus 5 exones contenidos en el cromosoma 2, generan repercusiones en su actividad enzimática. En el SCN tipo I se producen proteínas truncas o sin actividad, a diferencia del SCN tipo II en el cual las mutaciones sólo resultan en una disminución de la actividad catalítica de la enzima. Dada esta diferencia, los niveles de bilirrubina sérica orientan a distinguir entre los tipos de SCN, ya que cuando hay algo de actividad enzimática, la BT raramente supera los $20 \mathrm{mg} / \mathrm{dl}^{8}$. La actividad enzimática también puede evaluarse directamente en el tejido hepático ${ }^{4}$, o se puede medir la actividad de la enzima analizando los metabolitos producidos por ésta. Por lo tanto, la ausencia de conjugados bilirrubínicos en las sales biliares orientan a SCN tipo $\mathrm{I}^{16}$. Otra diferencia clínica es la respuesta al fenobarbital como inductor de la enzima: en los pacientes con SCN tipo II los niveles de bilirrubina se reducen en más de $25 \%$ al usar este fármaco, mientras que en los SNC tipo I no se aprecia respuesta ${ }^{9}$.

La confirmación diagnóstica se basa en el análisis genético. Las mutaciones se han encontrado en los exones 1, 2, 3, y 4. Estas pueden ser "missense", "nonsense", deleciones de nucletótidos, codones de "stop", corrimiento del marco de lectura y mutaciones del sitio de "splicing", resultando en enzimas truncadas o proteínas enteras no activas. En ausencia de diagnóstico genético se recomienda hacer la distinción usando la respuesta al fenobarbital y los niveles de bilirrubina en sales biliares? La Tabla 1 muestra las principales diferencias entre estos síndromes ${ }^{4,10}$.

El tratamiento médico del SCN I consiste en fototerapia intensa, habitualmente definida por al menos $12 \mathrm{~h}$ diarias ${ }^{11}$. En un estudio de 7 niños, 5 no requirieron trasplante y se mantuvieron sin secuelas neurológicas, con niveles de bilirrubina entre $15 \mathrm{a} 25 \mathrm{mg} / \mathrm{dl}^{4}$. A edades tempranas, este tratamiento parece ser suficiente, pero a medida que los pacientes aumentan en edad, comienza a ser cada vez menos efectivo, por una menor relación superficie/peso ${ }^{1}$. Por esto es común que con el paso del tiempo los pacientes desarrollen kernicterus, con complicaciones neurológicas que incluyen retraso mental, sordera, ataxia, convulsiones y hasta coma. La principal causa de desarrollo de kernicterus, sin embargo, sigue siendo la mala adherencia al tratamiento ${ }^{12,13}$, tal como se describe en este mismo caso. El único tratamiento definitivo sigue siendo el trasplante hepático y se debe intentar a edades tempranas, pues el desarrollo de kernicterus es aceptado como una contraindicación, no así la encefalopatía bilirrubínica leve, donde se recomienda que previo a considerar un trasplante, el nivel de bilirrubina debe mantenerse bajo $20,5 \mathrm{mg} / \mathrm{dl}$ con fototerapia .

El presente caso muestra una paciente de 
Tabla 1. Diagnóstico diferencial de la hiperbilirrubinemia indirecta

\begin{tabular}{|c|c|c|c|}
\hline & SCN tipo I & SCN tipo II & Gilbert \\
\hline Histología hepática & $\begin{array}{l}\text { Normal. Presencia ocasional de } \\
\text { tapones de bilis en canalículos }\end{array}$ & $\begin{array}{l}\text { Microscopía electrónica: } \\
\text { hipertrofia e hiperplasia del } \\
\text { REL, prominencia de Golgi }\end{array}$ & Normal \\
\hline Bilirrubina indirecta (mg/dl) & $20-50$ & $<20$ & $<3$ \\
\hline Pruebas hepáticas & Normales & Normales & Normales \\
\hline Bilirrubina directa en bilis & Ausente o muy escasos & $\begin{array}{l}\text { Predominio de } \\
\text { monoglucorónidos }\end{array}$ & $\begin{array}{l}\text { Predominio de } \\
\text { diglucorónidos }\end{array}$ \\
\hline Respuesta al fenobarbital & Nula & Parcial & Buena \\
\hline Herencia & Recesiva & Recesiva & Dominante \\
\hline Prevalencia & $\begin{array}{l}\text { Muy baja } \\
(0,6 \text { por millón habs })\end{array}$ & Baja & Alta $(8 \%)$ \\
\hline Pronóstico & Riesgo elevado de kernicterus & Riesgo mínimo de kernicterus & Excelente \\
\hline Actividad UDP-GT & Nula & $10 \%$ & $25-40 \%$ \\
\hline
\end{tabular}

19 años tratada exclusivamente con fototerapia artesanal domiciliaria, con buenos resultados iniciales. La aparición de kernicterus, manifestado por retraso mental leve y síndrome convulsivo, fue secundaria a suspensión del tratamiento por 2 meses. Sus niveles de bilirrubina han ido en incremento a través de los años y probablemente llegará un punto en que la fototerapia será insuficiente. En ella hubiese sido necesario plantear el trasplante hepático a una edad temprana. Sin embargo, en el contexto nacional de esos años no fue posible, dado el incipiente desarrollo del programa nacional de trasplante hepático ${ }^{14}$. La experiencia internacional es limitada: dos de 7 niños fueron trasplantados exitosamente en un estudio español ${ }^{4}$, y en el registro mundial se reportan 57 pacientes, con 21 trasplantados $(37 \%)$, sólo 15 (26\%) con daño cerebral en todo el registro (7 de ellos eran leves y se consideraron aptos para trasplante $)^{9}$. En dicho reporte, el más importante a nivel mundial, los pacientes con daño cerebral al momento del trasplante eran significativamente mayores que aquellos sin daño cerebral (14,3 vs 5,9 años).

Las guías internacionales requieren de un sistema de alta exigencia, con uso de una fuente de luz: 1) con un espectro dentro de espectro del rango de absorción de bilirrubina (400-520 nm); 2) un peak de emisión de $450 \pm 20 \mathrm{~nm}$; 3) un campo de irradiación que exponga al menos una superficie horizontal plana del paciente; y 4) un nivel de irradiación $\geq 30 \mu \mathrm{W} / \mathrm{cm}^{2} / \mathrm{nm}$ medido por un radiómetro ${ }^{15}$. No obstante, la implementación menos rigurosa de sistemas domiciliarios de fototerapia, como en nuestro caso, muestran éxito terapéutico siempre y cuando no se suspenda el tratamiento.

Este caso constituye un reporte de una enfermedad poco común en nuestro país y en el mundo, y muestra el enfoque diagnóstico y terapéutico en el contexto nacional de la atención de salud en un lugar lejano a la capital. Sin duda plantea nuevos desafíos, como el manejo de esta enfermedad por médicos especialistas no pediatras y la posibilidad de acceso a su tratamiento definitivo, el trasplante hepático.

Agradecimientos: Especialmente a la Dra. Jacqueline Jara P., del Hospital de Calbuco, por su colaboración en este trabajo.

\section{Referencias}

1. Van der Veere C, Sinaasappel M, McDonagh AF, Rosenthal $\mathrm{P}$, Labrune $\mathrm{P}$, Odièvre M, et al. Current Therapy for Crigler-Najjar Syndrome Type 1: Report of a World Registry. Hepatology 1996; 24 (2): 311-5.

2. Crigler J, Najjar V. Congenital familial non hemolytic jaundice with Kernicterus. Pediatrics 1952; 10, 169-80.

3. Burchell B, Coughtrie M, Jansen P. Function and regu- 
lation of UDP-glucuronosyltransferase genes in health and liverdisease: Report of the seventh international workshop on glucuronidation September 1993; Pitlochry, Scotland. Hepatology 1994; 20: 1622-30.

4. Lodoso B, Palomo E, Camarena C, Díaz MC, Hierro L, De la Vega A, et al. Síndrome de Crigler-Najjar: diagnóstico y tratamiento. An Pediatr (Barc) 2006; 65 (1): 73-8.

5. Sinaasappel M, Jansen PLM. The differential diagnosis of Crigler-Najjar disease, types 1 and 2, by bilepigment analysis. Gastroenterology 1991; 100: 783-9.

6. Cashore WJ. Kernicterus and bilirubin encephalopathy. Semin Liver Dis 1988; 8: 163-7.

7. Shimizu N, Mizutani M, Aoki T. A case of Crigler-Najjar syndrome type I: long survival with bilirubin adsorption and liver transplantation. No To Hattatsu 2005; 37 (4): 337-41.

8. Bosma PJ, Chowdhury JR, Bakker CTM, Gantla S, De Boer A, Oostra BA et al. The genetic basis of the reduced expression of bilirubin UDP-glucuronosyltransferase 1 in Gilbert's syndrome. N Engl J Med 1995; 333: 1171-5.
9. Jansen P. Diagnosis and management of Crigler-Najjar syndrome. Eur J Pediatr 1999; 158 [Supp 2]: S89-S94.

10. Carriel J, Castañares A. Hiperbilirrubinemia indirecta de origen genético: Informe de un caso de síndrome de Crigler-Najjar de tipo II. Arch Argent Pediatr 2010; 108 (4): e100-e104.

11. O'Reilly C, Dixon R. Crigler-Najjar syndrome: treatment at home with phototherapy. Scott Med J 1988; 33: 335-6.

12. Gartner LM, Herrarias CT, Sebring RH. Practice patterns in neonatal hyperbilirubinemia. Pediatrics 1998; 101: 25-31.

13. Meropol SB, Luberti AA, De Jong AR, Weiss JC. Home phototherapy: use and attitudes among community pediatricians. Pediatrics 1993; 91: 97-100.

14. Corporación del Trasplante. Total trasplantes hepáticos realizados en Chile 1993-2012. Disponible en http:// www.trasplante.cl/ [Consultado el 5 de mayo de 2013].

15. Bhutani VK, Cline BK, Donaldson KM, Vreman HJ. The need to implement effective phototherapy in resourceconstrained settings. Semin Perinatol 2011; 35: 192-7. 